Salavera Borás, C. \& Usán Supervía, P. (2017). Repercusión de las estrategias de afrontamiento de estrés en la felicidad de los alumnos de Secundaria. Revista Electrónica Interuniversitaria de Formación del Profesorado, 20 (3), 65-77. DOI: http://dx.doi.org/10.6018/reifop.20.3.282601

\title{
Repercusión de las estrategias de afrontamiento de estrés en la felicidad de los alumnos de Secundaria
}

\author{
Carlos Salavera Bordás ${ }^{1,2}$, Pablo Usán Supervía, \\ ${ }^{1}$ Facultad de Educación, Universidad de Zaragoza \\ ${ }^{2}$ OPICS Observatorio para la Investigación e Innovación en Ciencias Sociales, Universidad de \\ Zaragoza
}

\section{Resumen}

El objetivo de este estudio ha sido describir las estrategias de afrontamiento utilizadas por los adolescentes en las distintas situaciones de estrés que les suceden en su vida cotidiana, así como su repercusión en la felicidad subjetiva. Para ello, se evaluó a 1.402 alumnos/as de Enseñanza Secundaria (711 chicos y 691 chicas) de entre 12 y 17 años. Los resultados muestran como los chicos hacen más uso de estrategias de tipo no productivo, mientras las chicas buscan más el apoyo social. El modelo creado para la variable dependiente felicidad permite una estimación correcta del 92,9\% de los casos $\left(X^{2}=477.351 ; p<0.001 ; R^{2}=0.607\right)$. Los resultados indican como la percepción de la felicidad subjetiva es mayor entre quienes usan estilos de afrontamiento orientado a otros, mientras, el afrontamiento no productivo, incide en una menor felicidad subjetiva. Como principales conclusiones, señalar la necesidad de trabajar con los adolescentes las estrategias de afrontamiento con el grupo, un mejor manejo de las estrategias de carácter más introspectivo, así como el incremento y entrenamiento en el uso de las estrategias de afrontamiento activo y de soporte emocional.

\section{Palabras clave}

Estrategias; Afrontamiento de estrés; Felicidad; Estudiantes de Secundaria.

\section{Contacto:}

Carlos Salavera Bordás, salavera@unizar.es, Facultad de Educación, Universidad de Zaragoza, c/ Pedro Cerbuna, 1250009 Zaragoza. 


\section{Repercussion of stress coping strategies on Secondary Education students' happiness}

\section{Abstract}

This study aimed to describe the coping strategies that adolescents adopt in the different situations of stress that occur in their everyday lives, and how they affect their subjective happiness. It evaluated 1,402 students (711 males, 691 females) aged 12-17 years in Spanish Compulsory Secondary Education. The results show how males resort more to unproductive-type strategies, while females seek more social support. The model created for the dependent variable happiness gave a correct estimation for $92.9 \%$ of all cases $\left(X^{2}=\right.$ 477.351; $\left.p<0.001 ; R^{2}=0.607\right)$. The results indicated how the perception of subjective happiness was greater for those who used others-focused coping strategies. The unproductive coping results implied less subjective happiness. The main conclusions include having to work with adolescents on coping strategies with the group, dealing better with more introspective strategies, and training in and increasing the use of active coping strategies and emotional support.

\section{Key words}

Strategies; Stress coping; Happiness; Secondary students.

\section{Introducción}

Una de las épocas en la vida de la persona en las que más está sometido a situaciones de estrés es en la adolescencia, es un momento caracterizado por los cambios, complicado, con situaciones que debe de afrontar el adolescente sin tener todavía los recursos necesarios para superarlas con éxito. El estrés es una de las características que marca la adolescencia de nuestros jóvenes, siendo casi dos tercios los adolescentes que experimentan síntomas propios del estrés al menos una vez por semana. En este sentido, el estudio de las estrategias de afrontamiento del estrés adquiere cada vez más importancia. Conocer cómo afrontan los jóvenes sus problemas, el uso, manejo y empleo de estas estrategias es un tema importante tanto en el campo de la investigación (Frydenberg \& Lewis, 1996; Lazarus y Folkman, 1986; Lee, Seo, Lee, Park, Lee, \& Lee, 2017), como en el campo docente (Cingolani \& Castaneiras, 2016; Undheim \& Sund, 2017).

El estrés y las estrategias empleadas en gestionarlo por parte de los jóvenes, pueden determinar aspectos fundamentales en el propio desarrollo del sujeto y en su proceso de aprendizaje (Delahaij \& van Dam, 2016). Desde esta idea, el afrontamiento del estrés es considerado junto con las estrategias conductuales, cognitivas y emocionales, una parte más de la competencia psicosocial que van adquiriendo los jóvenes a lo largo de su vida, que se acentúa en la adolescencia, y que son utilizadas por los adolescentes para afrontar las demandas de la vida, conformando aspectos tan importantes para su funcionamiento como el autoconcepto, la autoestima, las habilidades sociales y relacionales o la resolución de conflictos (Frydenberg y Lewis, 1997). El afrontamiento, también interviene en las emociones, tanto en la atención, como en la comprensión y regulación de las mismas, ayudando en muchas ocasiones a resolver algunos de los problemas que las provocaron. Los jóvenes, necesitan aprender como afrontar de una manera eficaz y eficiente el estrés para desarrollarse de una forma saludable y feliz (Johnson y Johnson, 2002). 
Frydenberg y Lewis (1997) plantearon que las estrategias frente al estrés pueden ser agrupadas en seis estilos de afrontamiento: 1) Estrategias de afrontamiento activo, dirigidas a solucionar el problema, consistentes en trabajar para resolver la situación problema. Se realiza con las siguientes estrategias: concentrarse en resolver el problema, esforzarse y tener éxito, preocuparse; 2) Estrategias de afrontamiento no productivo, combinación de estrategias improductivas de evitación que muestra una incapacidad empírica para afrontar los problemas. Engloba las siguientes estrategias: no afrontamiento, reducción de tensión y autoinculparse; 3) Estrategias de distanciamiento en general, que se refieren al intento de negar el problema y alejarse de él. Lo forman las siguientes estrategias: ignorar el problema y reservarlo para sí mismo; 4) Estrategias de búsqueda de apoyo social y emocional, referido a las búsqueda de personas significativas, para la realización de actividades relajantes y/o físicas. Incluye las siguientes estrategias: invertir en amigos, buscar pertenencia, buscar diversiones y distracción física; 5) Estrategias de interpretación positiva del problema, afrontando el problema desde una visión positiva y optimista, con expectativas de que éste se puede resolver. En este apartado, se encontrarían las estrategias: hacerse ilusiones, buscar apoyo espiritual y fijarse en lo positivo; y 6) Estrategias de búsqueda de apoyo instrumental, con una búsqueda de ayuda y opinión de los demás, ya sean profesionales o personas cercanas. Estarían en este grupo: búsqueda de apoyo social, acción social y búsqueda de apoyo profesional. Frydenberg y Lewis (1997) afirman que es posible hacer una distinción entre afrontamiento general y específico, es decir el modo con el que el adolescente encara cualquier situación (general) y una problemática particular (específico). En este trabajo se hará referencia al afrontamiento general.

Unido a estas estrategias de afrontamiento del estrés, que aparecen de una manera más visible en la adolescencia, existe otro fenómeno en este período, la felicidad, que aunque es algo ampliamente buscado por el ser humano, se realiza de desigual forma, siendo la adolescencia uno de los momentos críticos en esta búsqueda. Se piensa que las personas felices tienen éxito a través de múltiples dominios de la vida (pareja, amigos, estudios, trabajo, salud, amor,... ). Quienes se perciben a sí mismo como felices responden de manera más fácil, adecuada y adaptativa a situaciones cotidianas principalmente, pero también a otras como el afrontamiento del estrés, la toma decisiones o la percepción e interpretación de situaciones sociales, tanto favorables como adversas (Lyubomirsky, King \& Diener, 2005).

De una manera más concreta y específica, se ha venido hablando de una felicidad subjetiva (Lyubomirsky y Lepper, 1999), constatando que hay personas que a pesar de situaciones difíciles o adversas, con poca suerte con la salud, el amor, el trabajo o los estudios se consideran felices; mientras, otros se consideran ellos mismos infelices aún cuando disfrutan de todo tipo de suertes y ventajas en su vida diaria.

En últimas fechas, se han incrementado los estudios sobre el bienestar subjetivo, siendo un constructo fundamental en éste, la felicidad subjetiva (Ortíz, Gancendo y Reyna, 2013; Vera-Villaroel, Celis-Atenas y Córdoba-Rubio, 2011), pudiéndose afirmar que felicidad y bienestar suelen asociarse con la salud física, mental y la creatividad, siendo factores protectores de cuestiones tan relevantes como la depresión y el suicidio (Moyano y Ramos, 2007). Dentro de ese constructo de bienestar subjetivo, la persona al medir su felicidad subjetiva examina aspectos de su vida y emite un juicio sobre ésta mediante juicios subjetivos (Pavot, Diener, Colvin y Sandvik, 1991).

El propósito de esta investigación era analizar la influencia de las distintas estrategias de afrontamiento del estrés en el nivel de felicidad subjetiva en estudiantes de $1^{\circ}$ a $4^{\circ}$ de Enseñanza Secundaria Obligatoria (ESO) teniendo en cuentas las siguientes 
hipótesis: $\mathrm{H} 1$ ) los chicos emplean de una manera distinta las estrategias de afrontamiento del estrés que las chicas; $\mathrm{H}_{2}$ ) las estrategias de afrontamiento de estrés de empleadas por los estudiantes de Secundaria inciden en su felicidad subjetiva.

\section{Metodología}

\section{Participantes}

El muestreo realizado fue de tipo estratificado siendo la unidad primaria la titularidad de los centros y la etapa de secundaria el nivel educativo $\left(3^{\circ}, 4^{\circ}\right.$ ESO y $1^{\circ}, 2^{\circ}$ Bachillerato). La muestra estuvo formada por 1402 estudiantes de $1^{\circ}$ a $4^{\circ}$ de ESO de seis Institutos públicos de Educación Secundaria: 711 chicos (50.71\%) y 691 chicas (49.29\%), que participaron en el estudio de manera voluntaria. La edad de los participantes fue de 12 a 17 años, con un media de 14.27 años (d.s. = 1.718). El estudio observó las consideraciones éticas de la Declaración de Helsinki y cumplió los criterios éticos de investigación con seres humanos (consentimiento informado de padres y madres, profesores y alumnos y derecho de información, protección de datos personales y garantías de confidencialidad, no discriminación, gratuidad y posibilidad de abandonar el estudio en cualquiera de sus fases). Realizado el cálculo de la representatividad de la muestra, con un nivel de confianza del $99 \%$ y un error muestral del $5 \%$, se obtuvo que la muestra final encuestada resultó representativa de la provincia de Zaragoza. El estudio se diseñó como un estudio retrospectivo ex - post facto (Montero y León, 2007).

Tabla 1.

Distribución de la muestra por edades $(n=1402)$

\begin{tabular}{ccc|ccc} 
Edad & Frecuencia & Porcentaje & Edad & Frecuencia & Porcentaje \\
\hline 12 & 218 & $15.55 \%$ & 15 & 352 & $25.10 \%$ \\
13 & 348 & $24.83 \%$ & 16 & 104 & $7.42 \%$ \\
14 & 354 & $25.25 \%$ & 17 & 26 & $1.85 \%$ \\
\hline
\end{tabular}

\section{Instrumentos}

Escala de Afrontamiento para Adolescentes (ACS) (Frydenberg \& Lewis, 1996):

Es un cuestionario de 80 ítems, 79 de tipo cerrado de tipo Likert de cinco puntos (no me ocurre nunca o no lo hago; me ocurre o lo hago raras veces; me ocurre o lo hago algunas veces; me ocurre o lo hago a menudo; me ocurre o lo hago con mucha frecuencia) y uno final abierto, permite evaluar 18 estrategias de afrontamiento: buscar apoyo social (SO), concentrarse en resolver el problema (RP), esforzarse y tener éxito (ES), preocuparse (PR), invertir en amigos íntimos (Al), buscar pertenencia (PE), hacerse ilusiones (HI), falta de afrontamiento o no afrontamiento (NA), reducción de la tensión (RT), acción social (SO), ignorar el problema (IP), autoinculparse (CU), reservarlo para sí (RE), buscar apoyo espiritual $(A E)$, fijarse en lo positivo (PO), buscar ayuda profesional (AP), buscar diversiones relajantes (DR) y distracción física (FI). Estas 18 escalas se agrupan en seis estilos básicos de afrontamiento: Afrontamiento activo, Afrontamiento no productivo, Distanciamiento en general, Búsqueda de apoyo social y emocional, Interpretación positiva del problema y Búsqueda de apoyo social instrumental. En el presente estudio, esta escala mostró una alta consistencia interna, alfa de Cronbach de 0.78 . 
Escala de la felicidad subjetiva (Lyubomirsky \& Lepper, 1999):

Es una medida global de felicidad subjetiva, que evalúa una categoría molar de bienestar como fenómeno psicológico global. Esta escala va más allá de la suma de estados emocionales positivos y negativos, y cogniciones relacionadas con el fenómeno, considerando la definición de felicidad desde la perspectiva de quien responde, asumiendo el supuesto que aún cuando existen diversos motivos para ser feliz, la mayoría de las personas tiene su propia idea de qué es ser feliz, cuando lo son o cuando no, siendo capaces de reportarlo (Lyubomirsky, 2008). Consta de 4 ítems con respuesta tipo Likert, su corrección se hace mediante la sumatoria de los puntajes obtenidos y se dividen en el número total de ítem. En este estudio, esta escala muestra una alta consistencia interna, con un coeficiente alfa de Cronbach de 0.82 .

\section{Procedimiento}

Para la selección de la muestra se solicitó la colaboración de los centros mediante llamada telefónica y, una vez confirmada la participación, se elaboró la lista de los centros participantes. En el momento de la entrega de cada escala, se procedió a explicar a los participantes el objetivo de la investigación, incidiendo en la importancia que tenía la cumplimentación de todos los ítems.

Los participantes tenían treinta minutos para completar los cuestionarios que se acaban de describir. En todos los casos se les recordaba que la información recogida era anónima y confidencial. Los datos recogidos en el presente estudio se obtuvieron entre los meses de abril y mayo de 2016.

Para el análisis estadístico de los datos se ha empleado el programa estadístico SPSS 22.0. Se realizó un análisis descriptivo para cada una de las variables. En todos los casos se trabajó con el menor nivel de significación posible y se han considerado significativas aquellas diferencias con un valor de $p<0.05$. Los contrastes se plantearon de forma bilateral. Finalmente, el establecimiento de ecuaciones predictoras en función de la felicidad, se realizó mediante la técnica de regresión logística, siguiendo el procedimiento de regresión por pasos hacia delante basado en el estadístico de Wald. Finalmente, con el programa estadístico AMOS v.24, se planteó un modelo de ecuaciones estructurales que permitiese validar y cuantificar las relaciones entre la felicidad subjetiva y las estrategias de afrontamiento de estrés utilizadas por los estudiantes de Secundaria.

\section{Resultados}

En las tablas 2 y 3 se presentan los resultados obtenidos por los participantes en la presente investigación. En primer lugar, el porcentaje de uso de las distintas estrategias adoptadas por los estudiantes varones y mujeres (Tabla 2), dividiéndose en cinco grupos, según el grado de utilización de la estrategia: 1) no utilizada; 2) raras veces; 3) algunas veces; 4) a menudo; 5) con mucha frecuencia. 
Tabla 2.

Análisis descriptivos por género de las estrategias de afrontamiento y la felicidad

\begin{tabular}{|c|c|c|c|c|c|c|c|c|c|c|}
\hline \multirow{2}{*}{$\begin{array}{c}\text { Estrategias } \\
\text { afrontamiento estrés }\end{array}$} & \multicolumn{5}{|c|}{ Chicos (en \%) } & \multicolumn{5}{|c|}{ Chicas (en \%) } \\
\hline & 1 & 2 & 3 & 4 & 5 & 1 & 2 & 3 & 4 & 5 \\
\hline Buscar apoyo social & 4.63 & 12.86 & 40.48 & 37.39 & 4.63 & 0.63 & 12.68 & $35 \cdot 52$ & 44.19 & 6.98 \\
\hline $\begin{array}{l}\text { Concentrarse en } \\
\text { resolver el problema }\end{array}$ & 1.03 & 9.26 & 36.88 & 43.22 & 7.2 & 1.27 & 8.88 & 41.23 & 41.65 & 6.98 \\
\hline Esforzarse y tener éxito & 0.51 & 7.55 & 26.93 & $49 \cdot 57$ & 14.92 & 0 & 8.25 & 27.27 & $54 \cdot 33$ & 10.15 \\
\hline Preocuparse & 0.51 & 6.17 & 37.39 & 43.4 & 12.52 & 0.63 & 3.81 & 30.44 & 56.45 & 8.67 \\
\hline $\begin{array}{l}\text { Invertir en amigos } \\
\text { íntimos }\end{array}$ & 3.6 & 16.3 & 42.88 & $29 \cdot 5$ & 7.72 & 1.9 & 15.86 & $44 \cdot 4$ & 30.87 & 6.98 \\
\hline Buscar pertenencia & 0 & 8.06 & 38.42 & 43.74 & 7.2 & 0.63 & 4.44 & 39.96 & 49.89 & 5.07 \\
\hline Hacerse ilusiones & 3.09 & $19 \cdot 38$ & 50.26 & 26.24 & 1.03 & 0.63 & 21.56 & 48.84 & 27.7 & 1.27 \\
\hline Falta de afrontamiento & $25 \cdot 56$ & 53.86 & 18.52 & 1.03 & 1.03 & 20.93 & 55.6 & 19.66 & 3.17 & 0 \\
\hline Reducción de la tensión & $43 \cdot 57$ & 46.14 & 8.23 & 2.06 & 0 & 30.23 & 51.37 & 11.42 & 6.34 & 0 \\
\hline Acción social & 16.3 & 62.78 & 16.47 & 4.46 & 0 & 26 & 61.95 & $9 \cdot 51$ & 2.54 & 0 \\
\hline Ignorar el problema & 18.87 & 45.11 & $25 \cdot 39$ & 9.78 & 0.86 & $25 \cdot 37$ & $47 \cdot 57$ & 26.43 & 0.63 & 0 \\
\hline Autoinculparse & 5.66 & 30.36 & 43.4 & $17 \cdot 5$ & 3.09 & 5.07 & 29.81 & 45.67 & 13.74 & $5 \cdot 71$ \\
\hline Reservarlo para sí & 2.57 & 31.05 & 45.28 & 18.52 & 2.57 & 6.34 & 38.05 & 37.84 & 13.95 & 3.81 \\
\hline Buscar apoyo espiritual & 10.46 & 50.94 & 27.27 & 9.26 & 1.03 & 8.25 & 56.87 & 22.2 & 12.68 & 0 \\
\hline Fijarse en lo positivo & 2.06 & 8.58 & $34 \cdot 31$ & 38.59 & 14.92 & 2.54 & 10.78 & 29.81 & 44.82 & 12.05 \\
\hline $\begin{array}{l}\text { Buscar ayuda } \\
\text { profesional }\end{array}$ & 9.09 & 32.25 & 33.1 & 20.41 & 5.15 & 10.78 & 40.59 & 36.58 & 8.25 & 3.81 \\
\hline $\begin{array}{l}\text { Buscar diversiones } \\
\text { relajantes }\end{array}$ & 1.03 & 9.26 & 14.24 & 42.54 & 32.42 & 0.63 & 10.78 & 13.95 & 48.2 & 26.43 \\
\hline Distracción física & 1.54 & 5.49 & 9.43 & 33.28 & 50.26 & 3.17 & 19.03 & 20.08 & 26 & 31.71 \\
\hline
\end{tabular}

Utilización de estrategias: 1) no utilizada; 2) raras veces; 3) algunas veces; 4) a menudo; 5) con mucha frecuencia

En cuánto al género (Tabla 2), se utilizan de forma diferente las estrategias de afrontamiento al estrés. Así, los chicos emplearon más estrategias de distanciamiento en general (ignorar el problema y reservarlo para sí), así como de búsqueda de apoyo social instrumental (acción social y búsqueda de apoyo profesional), mientras las chicas hicieron uso de la búsqueda del apoyo social y reducción de la tensión, no encontrándose diferencias en cuánto al uso de las estrategias de afrontamiento activo, interpretación positiva del problema, búsqueda de apoyo social y emocional y afrontamiento no productivo. En la felicidad subjetiva, no intervino la variable género $(F=.013 ; p=.908)$, pero sí la edad $(F=$ 93.474; $p<.001)$. Los resultados indican como las estrategias más utilizadas por los participantes (Tabla 3) están relacionadas con un afrontamiento activo (concentrarse en resolver el problema, esforzarse y tener éxito, preocuparse) y las estrategias de búsqueda de apoyo social y emocional (buscar pertenencia, buscar diversiones y distracción física), mientras que las estrategias menos utilizadas pertenecen al afrontamiento no productivo (falta de afrontamiento, reducción de la tensión). Se observó como la edad influye en un distinto uso de casi todas las estrategias de afrontamiento (excepto acción social y reservarlo para sí). 
Tabla 3.

Análisis descriptivos y comparativa de medias de las estrategias de afrontamiento y la felicidad

\begin{tabular}{lcccccc}
\multicolumn{1}{c}{ Estrategias afrontamiento estrés } & \multicolumn{3}{c}{ Género } & \multicolumn{2}{c}{ Edad } \\
\hline & $x$ & d.s. & $\mathrm{F}$ & Sig. & $\mathrm{F}$ & Sig. \\
Buscar apoyo social & 3.34 & .872 & 17.029 & .001 & 7.211 & .001 \\
Concentrarse en resolver el problema & 3.46 & .804 & .778 & .378 & 7.980 & .001 \\
Esforzarse y tener éxito & 3.69 & .800 & 1.506 & .220 & 22.043 & .001 \\
Preocuparse & 3.65 & .763 & 3.555 & .060 & 2.612 & .011 \\
Invertir en amigos íntimos & 3.24 & .903 & .465 & .495 & 4.932 & .001 \\
Buscar pertenencia & 3.53 & .723 & .386 & .534 & 12.055 & .001 \\
Hacerse ilusiones & 3.05 & .771 & 1.112 & .292 & 18.647 & .001 \\
Falta de afrontamiento & 2.01 & .746 & 2.631 & .105 & 7.976 & .001 \\
Reducción de la tensión & 1.80 & .769 & 37.110 & .001 & 11.617 & .001 \\
Acción social & 2.00 & .695 & 31.639 & .001 & 1.732 & .097 \\
Ignorar el problema & 2.17 & .849 & 35.404 & .001 & 4.947 & .001 \\
Autoinculparse & 2.83 & .905 & .582 & .446 & 7.299 & .001 \\
Reservarlo para sín & 2.80 & .874 & 11.849 & .001 & 1.858 & .073 \\
Buscar apoyo espiritual & 2.39 & .824 & .006 & .937 & 14.203 & .001 \\
Fijarse en lo positivo & 3.55 & .926 & .437 & .509 & 17.816 & .001 \\
Buscar ayuda profesional & 2.69 & .993 & 26.039 & .001 & 13.637 & .001 \\
Buscar diversiones relajantes & 3.93 & .995 & 2.145 & .143 & 2.806 & .007 \\
Distracción física & 3.98 & 1.111 & 114.610 & .001 & 8.813 & .001 \\
\hline
\end{tabular}

Además, se realizó una regresión múltiple (Tabla 4) para predecir el resultado de una variable categórica, seleccionando como variables predictoras las puntuaciones factoriales correspondientes a estrategias de afrontamiento y como variable criterio la felicidad. Este análisis es útil para modelar la probabilidad de un evento (felicidad) ocurriendo como función de otros factores (estrategias de afrontamiento), que era uno de los objetivos del estudio.

La tabla 4, muestra los pasos seguidos por los modelos en la introducción de las variables explicativas que han resultado significativas para la predicción de la probabilidad de felicidad. El modelo creado para la variable dependiente felicidad permite una estimación correcta del $92.9 \%$ de los casos $\left(X^{2}=477.351, p<0.001\right)$, entrando a formar parte de la ecuación las variables predictoras preocuparse, buscar pertenencia, hacerse ilusiones, falta de afrontamiento, reducción de la tensión, acción social, autoinculparse, reservarlo para sí mismo, buscar apoyo espiritual y fijarse en lo positivo. En este sentido, el estadístico $R^{2}$ de Nagelkerke estima un valor de ajuste de 0.607 para felicidad. Los componentes del modelo se expresan en la tabla 4. En cuanto a felicidad, las odds ratio obtenidas para esta variable indicaron que: a) la probabilidad de tener una percepción de felicidad subjetiva es 4.942 veces mayor entre los estudiantes de Secundaria que usan como estrategia buscar pertenencia; un 2.599 entre quienes emplean buscar apoyo espiritual; un 2.175 en los adolescentes que hacen uso de fijarse en lo positivo; y un 1.891 y un 1.622 entre quienes usan la acción social y hacerse ilusiones, como principales variables implicadas; b) las variables preocuparse, falta de afrontamiento, reducción de la tensión, autoinculparse y reservarlo para sí, inciden en una menor felicidad subjetiva. 
Tabla 4.

Resultados derivados de la regresión logística para la probabilidad de Felicidad

\begin{tabular}{lcccccc} 
& B & E.T. & Wald & $p$ & OR & IC 95\% \\
\hline Preocuparse & -.827 & .190 & 18.828 & .001 & .438 & $.301-.636$ \\
Buscar pertenencia & 1.598 & .232 & 47.494 & .001 & 4.942 & $3.137-7.784$ \\
Hacerse ilusiones & .484 & .180 & 7.236 & .007 & 1.622 & $1.140-2.308$ \\
Falta de afrontamiento & -1.007 & .211 & 22.670 & .001 & .365 & $.241-.553$ \\
Reducción de la tensión & -.445 & .158 & 7.889 & .005 & .641 & $.470-.874$ \\
Acción social & .637 & .238 & 7.168 & .007 & 1.891 & $1.186-3.016$ \\
Autoinculparse & -1.008 & .191 & 27.840 & .001 & .365 & $.251-.531$ \\
Reservarlo para sí & -.628 & .159 & 15.695 & .001 & .534 & $.391-.728$ \\
Buscar apoyo espiritual & .955 & .186 & 26.455 & .001 & 2.599 & $1.806-3.739$ \\
Fijarse en lo positivo & .777 & .147 & 28.028 & .001 & 2.175 & $1.631-2.900$ \\
\hline \multicolumn{2}{c}{ NOTA: B = coeficiente; E.T. = error estándar; $p=$ probabilidad; OR = odds ratio; } \\
\multicolumn{1}{c}{ IC 95\% = intervalo de confianza al 95\%. }
\end{tabular}

Por último, se intentó contrastar la hipótesis sobre la existencia de relaciones significativas entre la felicidad subjetiva y las estrategias de afrontamiento de estrés utilizadas por los estudiantes de Secundaria. En la Figura 1 se muestra el resultado del análisis con ecuaciones estructurales mediante el método de extracción de máxima verosimilitud, confirmando la adecuación del modelo compuesto por los dos constructos planteados en el estudio. El modelo indica una alta correlación entre la felicidad subjetiva y las estrategias de afrontamiento activo $(r=.70)$, las estrategias de búsqueda de apoyo social instrumental $(r=.69)$, las estrategias de interpretación positiva del problema $(r=.62)$, las estrategias de búsqueda de apoyo social y emocional $(r=.61)$, mientras las estrategias de afrontamiento no productivo $(r=-.16)$ y distanciamiento en general $(r=-.14)$ mostraron correlaciones inversas con la felicidad subjetiva. Por lo que se refiere al ajuste del modelo, los diversos índices de ajuste resultaron adecuados, por lo que se puede afirmar que el modelo propuesto acerca de la estructura factorial entre la felicidad subjetiva y las estrategias de afrontamiento es sustentable: $\chi^{2}(8)=16.720, p<0.001 ; \chi^{2} / g \mid=2.090 ; C F I=$ $0.98 ; \mathrm{NFI}=0.97 ; \mathrm{TLI}=0.95 ; \mathrm{RMSEA}=0.056, \mathrm{IC} 95 \%(0.015-0.093)$.

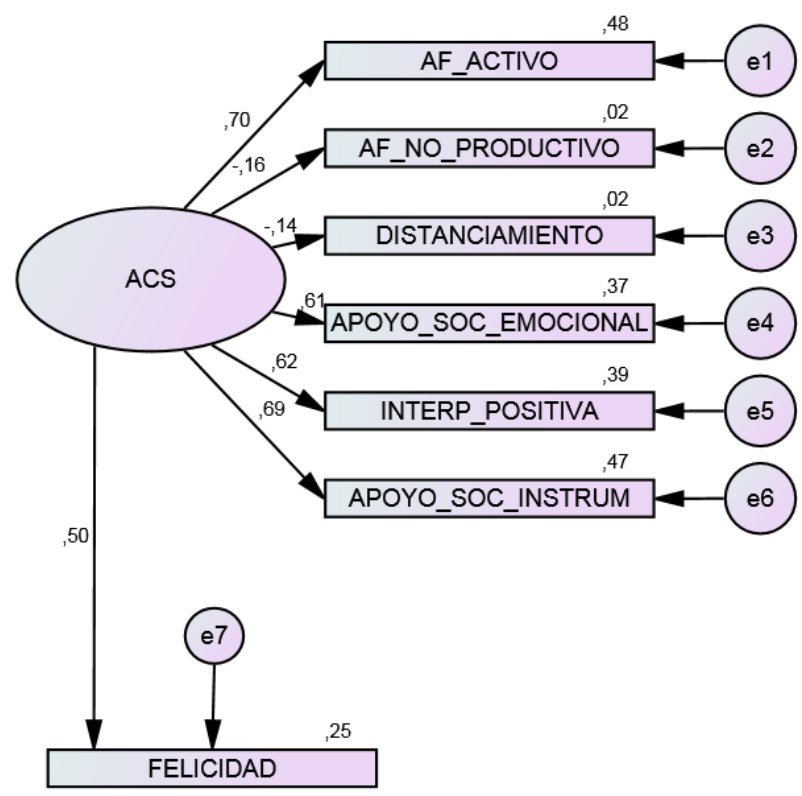

Figura 1. Modelo de ecuaciones estructurales entre la felicidad subjetiva y las estrategias de afrontamiento de estrés utilizadas por los estudiantes de Secundaria. 


\section{Discusión y conclusiones}

El propio enfoque de la Psicología Positiva señala como los diferentes recursos, estilos y estrategias de afrontamiento son importantes en la adaptación con éxito a las situaciones diarias de estrés (Compas, 1987; Seligman \& Csikszentmihalyi, 2000). Así, las estrategias de afrontamiento resultan útiles para un adecuado enfoque del problema generador del estrés, sobre todo cuando se trata de hacer frente a las emociones negativas asociadas a éste, siendo en estos casos, los adolescentes con peores estrategias de afrontamiento, los más afectados por una mala aceptación de ese estrés (Andrei, Mancini, Mazzoni, Russo \& Baldaro, 2015).

Los resultados de la presente investigación, señalan como la edad influye en la manera de utilización de las diferentes estrategias de afrontamiento, así como en la percepción de felicidad, disminuyendo ésta conforme aumenta la edad de los adolescentes, en línea con otros estudios anteriores (Viñas, González, García, Malo \& Casas, 2015). Esto puede deberse al paso de una visión optimista en la adolescencia temprana a una visión más pesimista ante el fracaso de los más mayores, con un cambio de expectativas que responde a su propia etapa evolutiva (Frydenberg, 1997). En cuanto al género, los chicos utilizan más estrategias de distanciamiento en general (ignorar el problema o reservarlo para sí) y de búsqueda de apoyo social instrumental (acción social y búsqueda de apoyo profesional), mientras las chicas hacen uso de la búsqueda del apoyo social y nos permite corroborar la primera hipótesis del estudio. Esto estaría en línea con investigaciones anteriores (Frydenberg y Lewis, 2009), y permite considerar el género como una variable que interviene en las estrategias de afrontamiento.

En cuanto a la felicidad, las estrategias de afrontamiento relacionadas con un estilo de afrontamiento en las que el adolescente busca la ayuda y la compañía de otros a la hora de afrontar las situaciones de estrés (buscar pertenencia, buscar apoyo espiritual, acción social) intervienen en determinar que los adolescentes tengan una mayor percepción de su felicidad subjetiva. Mientras, aspectos de un estilo de afrontamiento más introspectivo e individualizado (preocuparse, falta de afrontamiento, reducción de la tensión, autoinculparse y reservarlo para sí), indican como un mayor empleo de estas estrategias indican una menor felicidad subjetiva. Señalar en este sentido, que las estrategias pertenecientes a un estilo de afrontamiento activo (concentrarse en resolver el problema y esforzarse), así como otras más emocionales (distracción física, buscar diversiones relajantes) no intervienen en la percepción de la felicidad subjetiva. Los resultados nos llevan a afirmar el cumplimiento de la segunda hipótesis.

Se puede afirmar que los adolescentes, encuentran la felicidad en las estrategias que tienen relación con la ayuda del otro en la resolución de sus situaciones de estrés. Además, tener que afrontar los problemas por sí mismo, con estrategias que pueden exigirles un mayor esfuerzo a nivel personal e individual, está relacionado con una menor percepción de felicidad subjetiva en los adolescentes, en línea con otras investigaciones que explican la importancia de la felicidad individual en los colectivos (Uchida \& Oishi, 2016), mostrando el apoyo social y afrontamiento una asociación fiable con el bienestar durante la adolescencia (Zeidner, Matthews \& Shemesh, 2016). Esto, sin duda, debe hacernos reflexionar sobre el papel de la escuela en cómo se está educando a nuestros jóvenes, dado que éstos prefieren para su felicidad estrategias basadas en estilos de afrontamiento con el grupo frente a las estrategias de afrontamiento más individualizado. La escuela viene trabajando estos afrontamientos más personalizados en el sujeto, intentando dotar al estudiante de mecanismos propios de resolución de problemas que se le enseñan desde las aulas, cuando nuestros estudiantes prefieren estrategias basadas en el grupo y en la ayuda externa a la hora de afrontar sus situaciones de estrés, cuestión que puede venir 
relacionada con la autoestima y la capacitación como persona de nuestros alumnos. Tal y como plantean Matos, Camacho, Reis, Costa \& Galvão (2016) esto puede hacer ver a la escuela como "parte del problema" pero no "como parte de la solución", explicando en parte fenómenos como el bullying, en alumnos que no tienen las estrategias de afrontamiento individual necesarias para superar esas situaciones de estrés, pero tampoco, lo que es más importante para ellos, el apoyo del grupo para afrontar ese acoso. Que la estrategia que más determine la felicidad sea buscar pertenencia, es un buen indicador de este aspecto, dónde vemos que nuestros alumnos, son más felices perteneciendo a un grupo, que resolviendo por sí mismos sus problemas. Esto, lejos de ser un buen indicador, nos puede señalar que estamos ante un fenómeno de pertenencia gregaria por encima de las capacidades y aptitudes individuales de nuestros jóvenes. La investigación revela un aspecto muy preocupante a nivel del trabajo diario en nuestras aulas. El sistema educativo intenta implementar valores individuales y grupales en sus alumnos/as, sin embargo, los resultados de este estudio, muestran como los adolescentes, tienden a arropar sus decisiones y comportamientos en el grupo, mostrando cada vez menos necesidad de poner en marcha sus capacidades y recursos individuales, dejando sus decisiones en manos del grupo. Muchos de los constructos que conforman las habilidades interpersonales e intrapersonales de nuestros alumnos/as (autoeficacia, autoestima, habilidades sociales, resolución de problemas,... ) se ven diluidos en esa pertenencia al grupo, permitiendo a los adolescentes ocultarse en el grupo y poco a poco incapacitándole para resolución autónoma de sus problemas, con lo que esto puede suponer no sólo a nivel individual, sino también a nivel grupal y social para nuestros adolescentes en un futuro inmediato en su vida adulta, cuando tenga que tomar decisiones e interactuar no sólo a nivel grupal, sino en cuestiones con gran relevancia para su vida y desarrollo personal y profesional, pudiendo estar creando un mundo de adultos infelices ante su incapacidad de afrontar por sí mismos los problemas que les surjan en su vida diaria.

Como limitaciones del estudio, indicar que a pesar de lo amplio de la muestra, los resultados sugieren que aunque la felicidad y las estrategias de afrontamiento presentan una amplia relación, estamos hablando de felicidad subjetiva que induce una visión positiva sobre el afrontamiento emocional para futuros logros intermedios y no una visión positiva del futuro en general (Christophe \& Hansenne, 2016), por tanto limitada en el tiempo. En este punto, son necesarios más estudios e investigaciones que relacionen las estrategias de afrontamiento y la felicidad, con la edad y el género y que profundicen en esta línea. En cuanto a la edad, la realización de estudios longitudinales es necesaria para medir la evolución de las estrategias de afrontamiento en los adolescentes y su repercusión en la felicidad percibida en estas edades. En el caso del género, en línea por lo propuesto por Vantieghem, Vermeersch, \& Van Houtte (2014) son necesarios estudios que dentro de la investigación educativa, superen la brecha de género que tiende a centrarse en las diferencias entre los logros de los hombres y las mujeres, para trabajar en la asociación entre la identidad de género y las estrategias de afrontamiento de los adolescentes. Esto sin duda, dará una visión más pertinente sobre el manejo de las situaciones de estrés en los adolescentes, independientemente del género. Como vía de futuras investigaciones, y en línea de lo propuesto por distintos trabajos (Diener, Suh, Lucas \& Smith, 1999; Hampel \& Petermann, 2006), se debería profundizar en la interacción de los factores psicológicos con los diferentes acontecimientos vitales y seguir investigando la relación de las estrategias de afrontamiento del estrés con los diferentes componentes del bienestar subjetivo (felicidad subjetiva, satisfacción con la vida y afectos), así como en programas orientados a fomentar el uso de estrategias de afrontamiento activo que incrementen el bienestar personal en los adolescentes, contando para ello con la incorporación del profesorado y de las familias en esta tarea (Mirete, Soro y Maquilón, 2015; Silveira, 2016). 
Como principal conclusión, se puede determinar, en línea con lo propuesto por Graber, Turner \& Madill (2016), la necesidad de trabajar en el aula las estrategias de afrontamiento con el grupo, un mejor manejo de las estrategias de carácter más introspectivo, así como el incremento y entrenamiento en el uso de las estrategias de afrontamiento activo y de soporte emocional, mejorará el bienestar de los estudiantes de Secundaria y su percepción de la felicidad. Por otra parte, se debería trabajar desde los agentes implicados en la educación del alumnado, de una manera transversal en la capacitación de nuestros jóvenes para afrontar sus problemas por sí mismos, evitando apoyarse en movimientos gregarios y depositando esa responsabilidad en los otros, cuestión que va en detrimento de su propia percepción de la capacidad de afrontamiento de las situaciones de estrés. Por último, los resultados de la investigación nos animan a seguir buscando nuevas preguntas que nos ayuden a definir nuevas metodologías de trabajo y encontrar algunas respuestas para el afrontamiento del estrés, y que nos permitan avanzar en la construcción del desarrollo socioafectivo de los adolescentes.

\section{Referencias}

Andrei, F., Mancini, G., Mazzoni, E., Russo, P.M. \& Baldaro, M. (2015). Social status and its link with personality dimensions, trait emotional intelligence, and scholastic achievement in children and early adolescents. Learning and Individual Differences, 42, 97-105. http://dx.doi.org/10.1016/j.lindif.2015.07.014

Canessa, B. (2002). Adaptación psicométricos de las Escalas de Afrontamiento para Adolescentes de Frydenberg y Lewis en un grupo de escolares de Lima Metropolitana. Persona, 5, 191-233.

Christophe, V. \& Hansenne, M. (2016). Dispositional happiness and affective forecasting: General or specific effect? Journal of Positive Psychology, 11(2), 209-214. http://dx.doi.org/10.1080/17439760.2015.1058969

Cingolani \& Castaneiras, 2016). Coping strategies associated with emotional states in schooled adolescents. Acta Psiquiátrica y Psicológica de América Latina, 62(4), 222230.

Compas, B.E. (1987). Coping with stress during chilhood and adolescence. Psychological Bulletin, 101(3), 393-403.

Delahaij, R., \& Van Dam, K. (2016). Coping style development during military training: The role of learning goal orientation, and metacognitive awareness. Personality and Individual Differences, 92, 57-62. doi: 10.1016/j-paid.2015.12.012

Diener, E. (1984). Subjective well-being. Psychological Bulletin, 95(3), 542-575.

Diener, E., Suh, E.M., Lucas, R.E. \& Smith, H.L. (1999). Subjective well-being: Three decades of progress. Psychological Bulletin, 125(2), 276-302.

Frydenberg, E. (1997). Adolescent Coping. Theoretical and Research Perspectives. Nueva York: Routledge.

Frydenberg, E. \& Lewis, R. (1996). Escalas de afrontamiento para Adolescentes (ACS). [Adolescent coping scale]. Madrid: TEA Ediciones.

Frydenberg, E. \& Lewis, R. (2009). Relations among well-being, avoidant coping, and active coping in a large simple of australian adolescents. Psychological Reports, 104, 745758. http://dx.doi.org/10.2466/PRo.104.3.745-758

Graber, R., Turner, R., \& Madill, A. (2016). Best friends and better coping: Faciliting psychological resilience through boys' and girls' closest friendships. British Journal of Psychology, 2(1), 338-358. doi: 10.1111/bjop.12135 
Hampel, P. \& Petermann, F. (2006). Perceived stress, coping, and adjustment in adolescents. Journal of Adolescent Health, 38(4), 409-415.

http://dx.doi.org/10.1016/j.jadohealth.2005.02.014

Johnson, D. \& Johnson, R. (2002). Teaching students how to cope with adversity: the three Cs en Frydenberg, E. (Comp.). Beyond Coping. Meeting goals, visions, and challenges. Nueva York: Oxford University Press.

Lazarus, R. y Folkman, S. (1986). Estrés y procesos cognitivos. [Stress and cognitive processes]. Barcelona: Ediciones Martínez Roca.

Lee, J.H., Seo, M., Lee, M., Park, S.Y., Lee, J.H., \& Lee, S.M. (2017). Profiles of coping strategies in resilient adoldescents. Psychological Reports, 120(1), 49-69. doi: $10.1177 / 0033294116677947$

Lyubomirsky, S. \& Lepper, H. (1999). A measure of subjective happiness: preliminary reliability and construct validation. Social Indicators Research, 46, 137-155.

Matos, M.G., Camacho, I., Reis, M., Costa. \& Galvão, D. (2016). Worries, coping strategies and well-being in adolescents: highlights from HBSC study in Portugal. Vulnerable Children and Young Studies, 11(3), 274-280. http://dx.doi.org/10.1080/17450128.2016.1220655

Mirete, A.B., Soro, M., y Maquilón, J.J. (2015). El fracaso escolar y los enfoques de aprendizaje: medidas para la inclusión educativa. Revista Electrónica Interuniversitaria de Formación del Profesorado, 18(3), 183-196. doi: 10.6018/reifop.18.3.239021

Montero, I. y León, O. (2007). A guide for naming research studies in Psychology. International Journal of Clinical and Health Psychology, 7(3), 847-862.

Moyano, E. y Ramos, N. (2007). Bienestar subjetivo: midiendo satisfacción vital, felicidad y salud en población chilena de la Región Maule. [Well-being: measuring life satisfaction, happiness and health of the Chilean population Maule Region]. Universum, 22(2), 177-193.

Ortiz, M.V., Gancedo, K.M. y Reyna, C. (2013). Propiedades psicométricas de la escala de Felicidad Subjetiva en jóvenes y adultos de la ciudad de Córdoba-Argentina. Suma Psicológica, 20(1), 45-56. http://dx.doi.org/10.14349/sumapsi2013.1249

Pavot, W. G., Diener, E., Colvin, C. R., \& Sandvik, E. (1991). Further validation of the Satisfaction with Life Scale: Evidence for the cross-method convergence of wellbeing measures. Journal of Personality Assessment, 57, 149-161.

Seligman, M.E. \& Csikszentmihalyi, M. (2000). Positive psychology. An introduction. The American psychologist, 55(1), 5-14.

Silveira, H. (2016). La participación de las familias en los centros educativos. Un derecho en construcción. Revista Electrónica Interuniversitaria de Formación del Profesorado, 19(1), 17-29. doi: 10.6018/reifop.19.1.245511

Vantieghem, W., Vermeersch, H., \& Van Houtte, M. (2014). Transcending the gender dichotomy in educational gender gap research: The association between gender identity and academic self-efficacy. Contemporary Educational Psychology, 39(4), 369378. doi: 10.1016/j.cedpsych.2014.10.001

Vera-Villaroel, P., Celis-Atenas, K. y Córdoba-Rubio, N. (2011). Evaluación de la felicidad: análisis psicométrico de la Escala de Felicidad Subjetiva en población chilena. Terapia Psicológica, 29(1), 127-133. http://dx.doi.org/10.4067/S071848082011000100013

Viñas, F., González, M., García, Y., Malo, S. \& Casas, F. (2015). Los estilos y estrategias de afrontamiento y su relación con el bienestar personal en una muestra de adolescentes. Anales de Psicología, 31(1), 226-233. http://dx.doi.org/10.6018/analesps.31.1.163681 
Uchida, Y. \& Oishi, S. (2016). The Happiness of Individuals and the Collective. Japanese Psychological Research, 58(1), 125-141. http://dx.doi.org/10.1111/jpr.12103

Undheim, A.M., \& Sund, A.M. (2017). Associations of stressful life events with coping strategies of 12-15-year-old Norwegian adolescents. European Child and Adolescent Psychiatry, 1-11 (in press). doi: $10.1007 /$ s00787-017-0979-x

Zeidner, M., Matthews, G. \& Shemesh, D.O. (2016). Cognitive-Social Sources of Wellbeing: Differentiating the Roles of Coping Style, Social Support and Emotional Intelligence. Journal of Happiness Studies (in press). http://dx.doi.org/10.1007/s10902-015-9703-z 